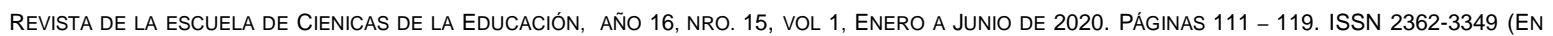
LÍNEA). LIDERAZGOS QUE INNOVAN CON TIC Y TRANSFORMAN LA ESCUELA. ROXANA NORA CARDARELLI. CARINA LION.

\title{
LIDERAZGOS QUE INNOVAN CON TIC Y TRANSFORMAN LA ESCUELA
}

\author{
Roxana Nora Cardarelli* \\ Universidad Torcuato Di Tella, Argentina \\ roxanacarda@gmail.com \\ Carina Lion** \\ Universidad de Buenos Aires, Argentina \\ carinalion@gmail.com
}

Recibido: 18/06/2019 Aceptado: 27/12/2019

\section{Resumen}

Esta investigación indagó acerca de las condiciones y las decisiones que conducen a directores/ coordinadores de instituciones educativas de distintos niveles educativos a incorporar tecnología en la gestión y a generar proyectos innovadores que superan modelos clásicos en el marco de la complejidad de los escenarios digitales contemporáneos. Se buscó triangular las relaciones entre liderazgo, innovación, gestión y TIC y la influencia de sus trayectorias, y se construyeron categorías analíticas acerca de los discursos, los usos y el sentido epistemológico que le otorga significación a la incorporación de TIC en sus proyectos. Se ha obtenido como resultado inédito una similitud paralela a las construcciones de procesos y formas de pensar con los que se vale el mundo del diseño multimedia, que necesariamente requieren trabajar en conjunto desde múltiples disciplinas.

Podemos destacar la utilización de metodologías de gestión cuyos patrones de comportamiento abordan etapas no lineales y se identifican con la metodología, DesignThinking así como también con arquitecturas de narrativas transmedia y creaciones de fandoms educativos entre otros mecanismos que nos mostraron liderazgos con capacidades y formas de pensar específicas para desarrollar innovaciones sustentables. Creemos que los resultados pueden generar un valioso aporte al sistema educativo para pensar en estilos de liderazgos y posibles procesos que invitan al desafío de gestionar con TIC en la escuela.

Palabras clave: Gestión - Liderazgo - Innovación - Tecnologías - Diseño multimedial.

\section{Abstract}

This research inquired about the conditions and decisions that lead directors / coordinators of educational institutions of different educational levels to incorporate technology into management. We

\footnotetext{
* Magister en Administración de la Educación y Especialista en Políticas Educativas , Universidad Torcuato Di Tella, Licenciada en Gestión de la Calidad Educativa, Universidad del Salvador, Profesora de Educación Preescolar, Instituto del Profesorado Sagrado Corazón. Ex Directora de Educación Municipal de la Municipalidad de Vicente López de la Provincia de Buenos Aires y ex Directora General de Desarrollo Social de la AGCBA, de la Ciudad Autónoma de Buenos Aires. Actualmente Coordinadora de Proyectos Pedagógicos Especiales en el Ministerio de Educación, Cultura, Ciencia y Tecnología de Argentina y representante en la Red Regional de Primera Infancia OEI, por Argentina.

* Doctora en Educación por la Facultad de Filosofía y Letras, Universidad de Buenos Aires. Especialista en Formación de formadores. Licenciada y Profesora en Ciencias de la Educación, Facultad de Filosofía y Letras, UBA. Profesora Adjunta de Educación y Tecnologías e Informática y Educación, Carrera de Ciencias de la Educación, FFyL, UBA. Profesora en Maestrías y Doctorados nacionales e internacionales.
} 


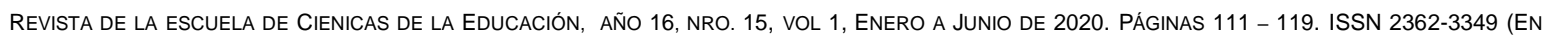
LÍNEA). LIDERAZGOS QUE INNOVAN CON TIC Y TRANSFORMAN LA ESCUELA. ROXANA NORA CARDARELLI. CARINA LION.

sought to triangulate the relationships between leadership, innovation, management and ICT and the influence of their trajectories. It has been obtained as an unprecedented result a parallel similarity to the constructions of processes and ways of thinking that the world of multimedia design uses, which necessarily require working together from multiple disciplines. From the consideration that managing also involves processing designs that work on devices for the construction of collaborative knowledge in multidisciplinary teams and the development of sustainable innovations, transmedia narratives, fandoms, generation of behavior patterns in non-linear stages (DesignThinking Methodology), among others. We believe that the results can contribute to revise the decisions and conditions for the inclusion of technologies in the educational system.

We can highlight the use of management methodologies whose behavior patterns address nonlinear stages and are identified with the methodology, DesignThinking as well as with transmedia narrative architectures and creations of educational fandoms among other mechanisms, showed us leaders with capacities to develop sustainable innovations. We believe that the results can generate a valuable contribution to the educational system to think about leadership styles and possible processes that invite the challenge of managing with ICT. at school.

Keywords: Management - Leadership - Innovation - Technologies - Multimedia Design.

\section{Introducción y Contexto}

El interés de indagar en los usos y discursos de las tecnologías de la Información y la Comunicación (TIC) en la gestión de la escuela surge porque las tecnologías se presentan hoy como medios de vida y comunicación, como uno de los lenguajes interactivos manifiestos de niños y jóvenes que van a un tipo de escuela. Escuela que particularmente se caracteriza por conservar en gran medida el formato o "gramática" que le dio origen como escuela "moderna" surgida y expandida hacia mediados del siglo XIX, como lo manifiestan Vincet y Lahire (2001); que necesita reconstruirse para compatibilizar con el tipo de alumnos que se recibe del contexto social, como sostiene Dubet (2002).

Sabemos que las TIC son parte de un mundo nuevo e interesante y también sabemos que cuesta moldear formatos fieles a génesis de otros siglos para chicos que devenían de otro tipo de mundo interesante; esto genera un estado de tensión que pone en discusión y abre interrogantes en torno a esta paradoja que puede o no otorgar posibilidades de nuevas formas de organización escolar que recrean la escuela. Edith Litwin (2008) nos llevó a pensar a la escuela en el contexto de la sociedad contemporánea desde lo político, social y cultural para que las propuestas pedagógicas resulten más significativas para los jóvenes y para que convivencia y labor educativa, sean escenarios de justicia y transformación social.

Esta investigación indagó centralmente distintas gestiones que utilizan TIC en forma sistemática; cabe destacar que se realizó desde un recorte que es la perspectiva de líderes que, a través de sus proyectos educativos y juntos a sus equipos de trabajo, lograron transformar la escuela y generar "innovaciones" en sus formas de interactuar, comunicarse, aprender y enseñar y el mismísimo significado de la palabra innovar, cuyo prefijo "in" figura penetración, está en, y el verbo "novo", "novas", "novare" que encarna hacer de nuevo, en este caso también con TIC.

Innovaciones que ofrecen cambios o rupturas de ciertas faces de la educación clásica conocida, como expresa Freire (1996) innovaciones decisivas que promueven comunidades de aprendizaje dialógico, educación para la comprensión, Perkins (2010) y pragmatismo democrático, Dewey (1996).

Se investigaron, específicamente, las relaciones que se establecen entre los discursos y los usos de la tecnología en la gestión escolar, así como también la incidencia de sus trayectorias personales y profesionales que influyen en sus liderazgos que transforman.

Los usos de las tecnologías en esta investigación refieren al hacer concretamente, al proyecto que los conduce a experimentar, a generar otras alternativas de organización, de oportunidades de aprender y de adjudicarse las TIC para su beneficio, y a interactuar también en entornos que superan los presenciales e invitan al pasaje asistemático de lo offline a lo online. Usos refiere a las innovaciones reales, no teóricas, que se están implementando, y se llevan a cabo con dinámicas de funcionamiento propias, con capacidades y recursos prácticos y viables de ser adquiridos e incorporados. Resulta importante destacar el sentido y la contextualización que los usos infieren en cuanto a la incorporación de la tecnología en la escuela, para desestimar la idea que utilizar TIC en la escuela implica innovar.

Los discursos refieren al decir, a la expresión subjetiva de dicha acción, de las necesidades, intereses y procesos con sus argumentos que les otorga sentido y los conduce a la selección, utilización, apropiación e interacción de las TIC en cada gestión, junto a las bondades y dificultades que ello implica en una organización.

Los interrogantes han sido: ¿Qué características presentan las gestiones que logran generar proyectos innovadores descriptos, entre otras cuestiones, por incorporar TIC y romper con formatos tradicionales de escuela? 


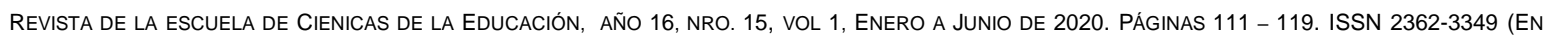
LÍNEA). LIDERAZGOS QUE INNOVAN CON TIC Y TRANSFORMAN LA ESCUELA. ROXANA NORA CARDARELLI. CARINA LION.

Estas preguntas derivaron a las siguientes: ¿Cuáles son las características del líder y sus equipos?, ¿Cuáles son sus formas de pensar que los movilizan?, ¿Qué necesidades y procesos transitan?, ¿Qué TIC utilizan y qué beneficios les aporta?, ¿Qué relaciones surgen del análisis de las respuestas a estos interrogantes? y ¿Qué tipo de trayectorias influyen en sus liderazgos innovadores?

En este sentido, resultó interesante analizar las relaciones que se producen entre liderazgo, innovación y gestión, la inclusión de la tecnología para la gestión, los procesos que transitan para liderar la gestión con tecnología e innovación; el tipo de pensamiento y su relación con el mundo de la tecnología y las relaciones que existen entre las trayectorias personales y el liderazgo innovador que los distingue.

Para dar respuestas a dichos interrogantes, se desarrolló un estudio exploratorio desde una temática de relevancia para la gestión de las escuelas y descriptivo en cuanto buscó identificar conexiones significativas de las relaciones entre los usos y discursos de las TIC en la gestión de la escuela, desde la perspectiva de directores/coordinadores investigados.

La investigación se estructuró desde el estado del arte y los antecedentes que obraron de punto de partida con las líneas de investigación y estudios sobre la incorporación de la tecnología en la gestión institucional, más el camino recorrido de Argentina de acceso e implementación de las TIC; y el marco conceptual donde se contextualizó a las TIC en los escenarios contemporáneos, a la gestión y cultura institucional, al rol del director/coordinador como líder de una institución educativa y de la innovación, y a los usos y discursos escolares en relación a las TIC. Los resultados del estudio se han obtenido desde:

1. El análisis de las concepciones generales de los directores/coordinadores entrevistados sobre liderazgo, gestión e innovación, el lugar de las TIC en la innovación y los proyectos innovadores junto a las herramientas que utilizan.

2. Las relaciones de los discursos con las tecnologías, lo que concierne a liderazgo y gestión, las perspectivas de equipo y las formas de actuar, sentir y relacionarse y a la relación entre discursos y liderazgos que descubre formas de pensar y actuar que se traducen en comportamientos que trascienden los ya conocidos de los directores o de quienes lideran gestiones escolares que se asimilan curiosamente a los del mundo multimedial.

3. Las necesidades y los procesos que conducen a incluir la tecnología para la gestión y la relación de liderazgo con innovación en la gestión, en donde la noción de tiempo, de espacios y estrategias ocuparon un lugar relevante junto a las formas de pensamiento que analizaron esta investigación.

4. El abordaje de la trayectoria personal del líder y su propia perspectiva que evidenciaron algunos disparadores e hitos posibles de ser tenidos en cuenta para lo que se espera de liderazgos escolares que innovan con TIC y transforman a la escuela. Además, se relacionaron las trayectorias y los recorridos personales con las actitudes cuestionadoras de supuestos establecidos y las tendencias a desestructurar supuestos naturalizados. Para ello se consideró las características de edad, de formación, de género y de contexto de trabajo. También las formas de pensarse a sí mismo y a sus trayectorias para reflexionar sobre usos y discursos.

5. Desde el plano epistemológico, optamos por un enfoque cualitativo; en lo metodológico, utilizamos el Método Comparativo Constante de Glaser y Strauss (1967) según el cual se estudió una muestra en relación con criterios teóricos hasta que dicha muestra saturó y dio cuenta de las tendencias más recurrentes, que permitieron una reconstrucción analítica desde lo que los autores denominan teoría fundada. La teoría fundada implica una interconexión entre la empírea y la teoría a través de un sistema categorial en el cual los datos dialogan con una interpretación conceptual.

Se generó teoría sobre la base de los puntos en común de los datos recolectados, para concluir con conceptos interrelacionados y no con enumeración de temas. Strauss y Corvin, (1990) sostienen la idea de generar teoría a partir de los datos que se recolectan durante el proceso de investigación y a la vez interrelacionarlos para seguir investigando.

Glaser y Strauss (1967/1999) plantean que cada incidente puede compararse con otros por sus propiedades o dimensiones para encontrar similitudes y diferencias, para determinar las categorías. Cuando las categorías o el sistema de categorías no dan más cuenta de rasgos preponderantes, satura, esto significa que por más que se continúe ampliando el muestreo, las evidencias serán las mismas. La instancia de saturación indica que la información que se obtiene no modifica las propiedades de las categorías ni sus relaciones. Para el armado de la muestra establecimos criterios precisos:

1. Líderes de proyectos de escuela de niveles educativos de educación formal: inicial, primario, medio y superior.

2. Líderes de escuelas reconocidas por enfoques que superan formatos tradicionales de escuela que utilizan TIC para gestionar y/o enseñar.

3. Líderes con experiencia en la gestión de cargos directivos y/o de coordinación escolar.

4. Líderes con proyectos reconocidos por premios obtenidos o resultados de los alumnos.

5. Líderes de distintas edades, género y formación. 


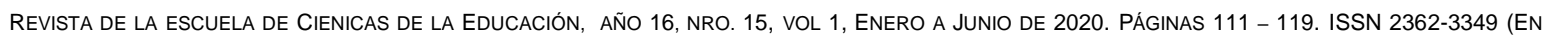
LÍNEA). LIDERAZGOS QUE INNOVAN CON TIC Y TRANSFORMAN LA ESCUELA. ROXANA NORA CARDARELLI. CARINA LION.

Utilizamos la entrevista como principal herramienta para la recolección de la información, para que desde la interacción social entre el entrevistador y el entrevistado, este último logre desenterrar de la memoria parte de su vida y de su experiencia (Sautu, 2003).

También se utilizaron como fuentes, entornos virtuales, sitios web y artículos de diario que dieron cuenta de sus proyectos innovadores y posicionamientos institucionales, para ampliar la comprensión de lo expresado por los entrevistados. Como reconoce Vasilachis de Gialdino (2006), el análisis de cómo las personas perciben las cosas no puede ignorar la importancia de cómo las hacen.

Se utilizaron entrevistas de tipo abierta y flexible desde un punteo preliminar se dio lugar a profundizar los temas que el propio entrevistado consideró interesante aportar.

A medida que se fue analizando la información y generando las categorías, más las relaciones entre las mismas, en el ir y venir de los testimonios, comenzó a reiterarse y a saturar la muestra en la séptima entrevista, por lo que la muestra se valió de siete entrevistados.

Para validar dichos discursos y chequear coherencias, contradicciones, vacíos, etc. con los usos y sus resultados, se generó una triangulación de la información en términos de Flick (1998), para:

a) Combinar perspectivas teóricas y metodológicas.

b) Enriquecer el análisis del objeto de la investigación.

c) Utilizar más de una aproximación en la recolección de la información para garantizar la validez de los datos y su confiabilidad.

d) Realizar un reconocimiento crítico del liderazgo en relación con la gestión, a partir de los tipos de pensamiento que los impulsan las interacciones y procesos que llevan a cabo y las trayectorias que los caracterizan.

Para el análisis, la interpretación y la construcción de categorías se abordó el enfoque metodológico: Grounded Theory - Método comparativo constante. Las entrevistas fueron grabadas y desgravadas en transcripciones textuales. Mediante el análisis posterior de las desgrabaciones y de la lectura de los sitios web, se reconocieron una serie de características relativas a las visiones de los entrevistados en relación a los procesos identificados y sus formas de pensamiento con los que se conducen; se construyeron las categorías de acuerdo con los pasos a seguir de dicha teoría. Se identificaron palabras y párrafos textuales claves para clasificarlos según el objetivo de investigación correspondiente, se buscaron las palabras y conceptos claves en común entre los entrevistados que al anticipar las posibles categorías específicas de análisis con sus cualidades desde su análisis interpretativo surgió una parte de los resultados de esta investigación. Luego se procedió a relacionar las categorías entre sí, lo que dio lugar al pasaje de un segundo nivel de categorías y de interpretación, esta vez integrando toda la información para avanzar en la triangulación, como plantea Denzin (1970) y posterior teorización.

\section{Resultados y conclusiones}

Las mediaciones tecnológicas que favorecen y enriquecen las posibilidades de proyectos innovadores en las escuelas nos permiten imaginar escenarios y entornos educativos que promueven y favorecen el desarrollo de nuevas oportunidades de aprendizaje. Diseñar, desde una forma de pensar particular de un líder la construcción de este tipo de escenarios y entornos de innovación en la gestión implica reconocer y reconstruir conceptualmente los procesos, dimensiones y significados que se despliegan en contextos singulares y complejos.

La principal y más contundente conclusión de esta investigación en relación con los liderazgos investigados y sus gestiones innovadoras con TIC es que el pensamiento que rige la acción y los modos de obrar de líderes no sólo en la gestión escolar sino también en sus propias vidas, tienen formas de pensar y abordajes en las construcciones de procesos equitativos con los que se vale el mundo del diseño multimedial; por lo que gestionar innovación con Tic en la escuela se puede traducir en "diseñar formas de operar con usuarios clave", que comienza por la impronta de quién asume el liderazgo pero trasciende con la tracción de un equipo que a su vez se convierte en líder y opera con las formas de pensar visibles en los hechos y las narrativas. Dicha relación de semejanza, se sintetiza en el siguiente cuadro:

Relaciones de semejanza

Formas de pensamiento/ procesos: Líderes investigados

(usos, discursos y trayectorias)

Teoría/procesos: Diseño multimedial 
ReVISTA DE LA ESCUELA DE CIENICAS DE LA EdUCACIÓN, AÑo 16, NRO. 15, VOL 1, ENERO A JUNIO DE 2020. PÁGINAS 111 - 119. ISSN $2362-3349$ (EN LÍNEA). LIDERAZGOS QUE INNOVAN CON TIC Y TRANSFORMAN LA ESCUELA. ROXANA NORA CARDARELLI. CARINA LION.

\begin{tabular}{|c|c|}
\hline Trabajo/líderes en equipo. & Trabajo en equipo (Metodología DesignThinking). \\
\hline $\begin{array}{l}\text { Pensamiento proyectual: empatiza y les abre } \\
\text { camino para inventar patrones. }\end{array}$ & $\begin{array}{l}\text { Generación de prototipos: Etapas no lineales: Empatiza, } \\
\text { define, idea, prototipa, testea (Metodología } \\
\text { DesignThinking). }\end{array}$ \\
\hline Escucha atenta. & $\begin{array}{l}\text { Para el diseño es mejor escuchar no al segmento que } \\
\text { tenga la gran mayoría de consumidores sino a los } \\
\text { usuarios clave. }\end{array}$ \\
\hline $\begin{array}{l}\text { Los resultados generan ambientes virtuales, } \\
\text { comunidades participativas, que incorporan lo } \\
\text { lúdico al trabajo y la colaboración en } \\
\text { comunidades de conocimiento específico. }\end{array}$ & $\begin{array}{l}\text { Los resultados pueden generar fandoms (colaboración en } \\
\text { comunidad). }\end{array}$ \\
\hline $\begin{array}{l}\text { Pensamiento lateral: Distintas alternativas de } \\
\text { resolver problemas. }\end{array}$ & $\begin{array}{l}\text { El diseño multimedial se enfoca en resolver los } \\
\text { problemas a los usuarios desde distintos ángulos. }\end{array}$ \\
\hline $\begin{array}{l}\text { Los resultados ofrecen testimonios que se } \\
\text { observan y reconstruyen in situ en nuevos } \\
\text { proyectos. }\end{array}$ & $\begin{array}{l}\text { Los resultados convergen en narrativas y re lecturas, las } \\
\text { narrativas transmedia nos suben al mundo de las } \\
\text { plataformas mediáticas. }\end{array}$ \\
\hline $\begin{array}{l}\text { Los resultados generan comunidades de } \\
\text { aprendizaje para el trabajo en red. }\end{array}$ & $\begin{array}{l}\text { Cultura de la convergencia: el consumo es una práctica } \\
\text { en red. }\end{array}$ \\
\hline $\begin{array}{l}\text { Los resultados generan nuevos formatos para } \\
\text { enseñar y relacionarse en forma } \\
\text { interdisciplinaria. }\end{array}$ & Cosmopedia. Construcción colectiva. \\
\hline
\end{tabular}

\section{Cuadro 1: Elaboración propia}

Las similitudes en las construcciones de procesos y formas de pensar de los líderes investigados en relación con los que se vale el universo del diseño multimedial nos aportan hitos a tener en cuenta para la gestión escolar.

Resulta importante tener en cuenta que el término multimedia se utiliza para referirse a cualquier objeto o sistema que utiliza múltiples medios de expresión (físicos o digitales) para presentar o comunicar información. También que el ámbito multimedial requiere necesariamente el trabajo conjunto desde diferentes disciplinas.

\section{Hitos a tener en cuenta para la gestión}


1. El líder innovador, piensa, se posiciona y gestiona desde una visión proyectual que empatiza con los otros y les abre el camino para inventar patrones, explorar nuevas posibilidades en forma individual y en equipo.

2. El liderazgo innovador asume al equipo como líderes del proyecto.

3- Líder +equipo +TIC son una tríada que sincroniza una gestión particular, cuya tracción rige proyectos entusiastas que desarrollan con equipos que supieron adquirir capacidades frente a determinadas necesidades

4- El pensamiento que rige la acción; el tipo de trato en el que se incluye también al virtual; las emociones positivas y las destrezas que desarrollan con TIC, son significativas a la hora de posicionarse frente a la acción con diversidad de enfoques alternativos que conquistan y avanzan sobre lo ya conocido.

5- La incorporación de TIC en la gestión escolar requiere de procesos específicos que varían por móviles y necesidades inherentes a los propósitos de cada gestión y su contexto.

6- El comienzo de los procesos en la incorporación de TIC suele desarrollarse por necesidad de facilitar la gestión administrativa y comunicacional y continúa por necesidad de relacionarse e interactuar en conjunto en redes escolares de manera más eficiente, a la vez que genera la formalización de los procesos necesarios para reflexionar la gestión.

7- El tiempo y el contexto son valores visibles y determinantes que motorizan la gestión proactiva, creativa y estratégica; aportan espacio y oportunidad para tomar decisiones y conceden o no, la ocasión de operar cambios para avanzar sobre las costumbres y hábitos naturalizados de la escuela. El tiempo pasado se presenta con aportes para combinar con el presente y oficiar nuevas formas de gestión que se traducen en presente y posible futuro innovador.

8- Los ambientes y entornos virtuales que involucran a las TIC cobran vital importancia, se presentan como motivadores de gestiones que innovan con nuevas propuestas para comunicar y relacionarse, en donde también la imagen adquiere protagonismo. Plataformas virtuales grupales e individuales, entornos participativos y aplicaciones comunicativas y de enseñanza, centran relevancia y ocasionan la oportunidad de generar fandoms educativos que se definen por su propia afinidad.

\section{Cuadro 2. Elaboración propia}

Al relacionar las trayectorias y los recorridos personales con sus liderazgos y formas de pensarse a sí mismo, desde sus discursos, obtenemos que:

Hitos a tener en cuenta para la gestión escolar 


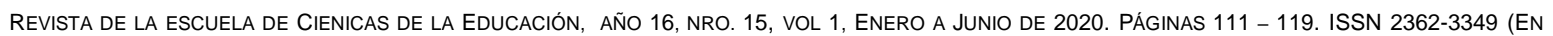
LÍNEA). LIDERAZGOS QUE INNOVAN CON TIC Y TRANSFORMAN LA ESCUELA. ROXANA NORA CARDARELLI. CARINA LION.

La trayectoria personal de un líder y sus modos de pensarse a sí mismo se relaciona en forma directa con el tipo de gestión que agencia, más allá de las características de edad, género, formación y contexto.

La actitud crítica al sistema se manifiesta a través de la preponderancia de las TIC como oportunidad para avanzar sobre estructuras atemporales.

La confluencia de las trayectorias de un líder innovador y las formas de pensamiento en la acción con TIC, pueden generar nuevas formas de organización para la gestión, nuevos planes de formación, formatos escolares alternativos con inclusive la incorporación de distintos enfoques pedagógicos, propuestas educativas interdisciplinarias en respuesta a características culturales.

Formas de pensamiento lateral y proyectual pueden conducir a innovar, a posibilitar abandonar lo disciplinar y realizar comprensiones interdisciplinarias para integrar conocimientos y abordajes de dos o más disciplinas para crear productos, plantear interrogantes solucionar problemas y dar explicaciones al mundo que los rodea, a la vez de visibilizar procesos.

Las trayectorias de los líderes investigados se caracterizan por superar formas de operar de la época y sus reacciones a contextos adversos se traducen más que en luchas colectivas, en actitudes resilientes.

Las preferencias de los líderes a la hora de elegir referentes y lecturas académicas trascienden lo cercano a su disciplina y se orientan al mundo de la tecnología y el diseño multimedial.

El pensamiento visible de los líderes conduce al aprendizaje en equipo de todos los protagonistas del cambio.

\section{Cuadro 3: Elaboración propia}

Si bien esta investigación partió en tiempo presente de los discursos de diseños y construcciones concretas de gestión que innova con TIC, dejó abierta la oportunidad de retomar la mirada en las mismas gestiones, cuando los líderes investigados ya no sean sus protagonistas, para analizar la sustentabilidad de los proyectos de innovación a través del tiempo a la par de sus procesos de reconstrucción para avanzar en sus proyectos. Y a partir de esta inquietud, plantear probables interrogantes como:

a) ¿La fuerza del dispositivo escolar de escuela clásica o tradicional, se impuso nuevamente? y/o ¿persisten con el paso del tiempo, las gestiones con formatos alternativos con TIC?

b) ¿Las gestiones con formatos alternativos con TIC avanzan en innovación?

c) ¿Qué factores, formas de pensar y obrar, influyen en la reconstrucción o retrocesos, si lo hubiere? Para, de esta manera, continuar la indagación sobre estas nuevas formas de organización que recrean la escuela y comprender si rompen o no con la paradoja del estado de tensión que las TIC generan en este 


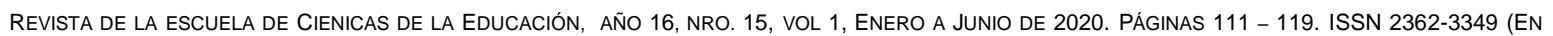
LÍNEA). LIDERAZGOS QUE INNOVAN CON TIC Y TRANSFORMAN LA ESCUELA. ROXANA NORA CARDARELLI. CARINA LION.

mundo que requiere poner en juego habilidades y conocimientos específicos que demanda la sociedad, también a través de la escuela.

Referencias bibliográficas

Aguerrondo, I. y Xifra, S. (2002). La escuela del futuro: cómo planifican las escuelas que innovan. Buenos Aires: Papers Editores.

Área, M. (2002). La integración escolar de las nuevas tecnologías. Entre el deseo y la realidad. Organización y Gestión Educativa. (6) p. 14-189.

Austin, K. (2003). Steal Like an Artist: 10 Things Nobody Told You About Being Creative. México: Santillana.

Bass, B. (1985). Leadership and performance beyond expectations. New York: Free Press

Bass, B. y Avolio, B. J. (1995). The Multifactor Leadership Questionnaire (MLQ). Redwood City: Mind Garden

Bass, B. (2000). El futuro del liderazgo en las organizaciones que aprenden. Bilbao: ICE de la Universidad de Deusto. Mensajero.

Blejmar, B. (2009). Gestionar es hacer que las cosas sucedan. Competencias, actitudes y dispositivos para diseñar instituciones. Buenos Aires: Noveduc.

Berger, P. y Luckmann, T. (1968). La Construcción social de la realidad. Buenos Aires: Amorrortu.

Bois Mansilla, V. y Gardner, H. (2006). Enseñar para la comprensión en las disciplinas y más allá de ellas. Tomado de Teaching for Understanding-within and across the Disciplines, Educational Leadership (February 1994). Recuperado de http://latitud-nodosur.org/IMG/pdf/EpCyDisciplinas.pdf

Bolivar, A. (2000). Los centros educativos como organizaciones que aprenden. Promesas y realidades. Madrid: Muralla.

Brown, T. (2008). Design thinking. Harvard: Business review.

Bruner, J. (1998a). Actos del significado: más allá de la revolución cognitiva. Madrid: Alianza.

Bruner, J. (1998b). Realidad mental y mundos posibles: los actos de la imaginación que dan sentido a la experiencia. Barcelona: Gedisa.

Bruner, J. (2000). Escenarios de Futuro. Nuevas Tecnologías y Sociedad de la Información; PREAL, Santiago de Chile, Documento de Trabajo № 16, 2000. Recuperado de http://www.preal.cl/brunner16.pdf

Burbules, N. C. y Callister, T. A. (2001). Riesgos y promesas de las nuevas tecnologías de la información. Buenos Aires: Granica.

Carbonel, J. (2001). La aventura de innovar. El cambio en la escuela. Madrid: Morata.

Castells, M. (2006). La Sociedad Red. Madrid: Alianza Editorial.

Covey, S. (2004). Los 7 hábitos de la gente altamente efectiva: la revolución ética en la vicia cotidiana y en la empresa. Buenos Aires: Paidós.

Cuban, L. (2001). Oversold and Underused: Computers in the Classroom. London: Harvard University.

De la Teja, I.; Lundgren-cayrol, K; Ganesan, R. y Spector, M. (2003). An Introduction to issues in the evaluation of Educational Technology: International Perspectives. Evaluation and Program Planning, 26,163-168.

de Bono, E. (1970). El pensamiento lateral. Manual de creatividad. Buenos Aires: Paidos.

Dewey, J. (1916). Democracy and Education. New York: Macmillan.

Denzin, N. (1970). Sociological Methods: a source Book. Chicago: Aldine Publishing Company.

Dubet, F. (2002). El declive de la institución: Profesiones, sujetos e individuos ante la reforma de Estado. Barcelona: Gedisa.

Dubrin, A.; Dalglish, C. \& Miller, P. (2006). Liderazgo 2. Dakota del Norte: Asia-Pacífico Ediciones.

Escudero, J. (1992). La integración escolar de las nuevas tecnologías de la información. Info-

Flavell, J. (1979). Metacognition and Cognitive Monitoring. A New Area of cognitive-developmental Inquiry. American: Psychologist.

Flick, U. (1998). An introduction to qualitative research. Londres: Sage.

Fernández, M. y Valverde, J. (2014). Comunidades de práctica: un modelo de intervención desde el aprendizaje colaborativo en entornos virtuales. Comunicar, 21 (42), p.97-105.

Freire, P. (1996). Pedagogía de la autonomía. Saberes necesarios para la práctica educativa. México: Siglo XXI.

Fullan, M. (2002). Los nuevos significados del cambio en la educación. Barcelona: Octaedro.

Fullan, M. (2016). La Dirección Escolar. Tres claves para maximizar su impacto. Madrid: Morata.

Gergen, K. (2006). El yo saturado. Dilemas de identidad en el mundo contemporáneo. Barcelona: Paidos.

Glaser, B. y Struss, A. (1967). El desarrollo de la teoría fundada. Chicago: Illinois: Aldine.

Goodson, I. G. y Mangan, J. M. (1996). Computer Literacy as Ideology en British Journal of Sociology of Education. (17), p. 4. Recuperado de www.ivorgoodson.com/s-Computer\%20Literacy-2

Haraway, D. (2004). Manifiesto para cyborgs. Ciencia, tecnología y feminismo socialista a finales del siglo XX. Valencia: Eutopía

Hargreaves, A. (1994). Changing teachers, changing times: teachers' work and culture in the postmodern age. London: Cassell. (Trad. cast.: Profesorado, cultura y postmodernidad. Madrid: Morata.

Hartle, F. y Hobby, R. (2003). Liderazgo en una comunidad de aprendizaje; Tu trabajo nunca volverá a ser el mismo. Valencia: Eutopía

Hately, N. \& Schiller, J., (2003). ¿Qué habilidades personales de las TIC necesitan los líderes escolares? Barcelona: Paidós.

Informe Kelburn, (1997). En: Martín, E. (2006). Tendencias y debates en la integración de las TIC al sistema educ ativo. La integración de las tecnologías de la información y la comunicación en los sistemas educativos. Capítulo 1. IIPEUNESCO.

Jenkins, H. (2008). La cultura de la convergencia de los medios de comunicación. Barcelona: Paidós.

Latour, B. (1999). Pandora's Hope: Essays on the Reality of Science Studies. Boston: Harvard Univ.

Lévy, P. (2004). La Inteligencia colectiva. Washington, DC: Biblioteca virtual Em saúde. 
ReVISTA DE LA ESCUELA DE CIENICAS DE LA EdUCACIÓN, AÑo 16, NRO. 15, VOL 1, ENERO A JUNIO DE 2020. PÁGINAS 111 - 119. ISSN $2362-3349$ (EN LÍNEA). LIDERAZGOS QUE INNOVAN CON TIC Y TRANSFORMAN LA ESCUELA. ROXANA NORA CARDARELLI. CARINA LION.

Litwin, E. (2005). Las nuevas tecnologías en tiempos de Internet. Buenos Aires: Amorrortu.

Litwin, E. (2008). El oficio de enseñar. Condiciones y Contextos. Primer capítulo: Escenas y experiencias en contextos reales. Buenos Aires: Paidós.

Litwin, E. (2008). Entiende a la innovación. Buenos Aires: Paidós

Maggio, M. (2012). Enriquecer la enseñanza. Los ambientes con alta disposición tecnológica como oportunidad. Buenos Aires: Paidós.

Means, B., \& Olson, K. (1995). Technology's Role in Education Reform:Findings from a National Study of Innovating Schools. Menlo Park, CA: SRI International. Recuperado de http://www.ed.gov/pubs/EdReformStudies/EdTech/

Mezzadra, F. y Bilbao, R.2010). Las nuevas tecnologías de la información y la comunicación en educación: discusiones y opciones de política educativa. Buenos Aires: Fundación CIPPEC.

Palamidessi, M. (2006). La escuela en la sociedad de redes. Una introducción a las tecnologías de la información y la comunicación en la educación. Buenos Aires: Fondo de Cultura Económica.

Ortiz, M. y Lobato, X. (2003). Escuela inclusiva y cultura escolar: algunas evidencias empíricas. Bordón, 55 (1), pp. 27-39.

Owen, C. (2007). Design thinking: Notes on its nature and use en Design Research Quarterly. (21), pp. 16-27.

Perkins, D., (2001). La persona-más: una visión distribuida del pensamiento y del aprendizaje. Buenos Aires: Amorrortu.

Reig, D. y Vílchez, L. F. (2013). Los jóvenes en la era de la hiperconectividad: tendencias, claves y miradas. Madrid: Fundación Telefónica y Fundación Encuentro.

Rivas, A.; André, F. y Delgado, L.E. (2017). 50 Innovaciones educativas para escuelas. Buenos Aires: Santillana.

Rivas Navarro, M. (2000). Innovación educativa. Teoría, proceso y estrategias. Madrid: Síntesis.

Rothschild, W. (1993). Las cuatro caras del liderazgo estratégico: líderes audaces, cautelosos, cirujanos y funerarios. Buenos Aires: Macchi.

Sautu, R. (2003). Todo es teoría. Buenos Aires: Lumiere.

Schein, E. (1997). Psicología de la Organización. 3a.ed.Mexico DF: Prentice-Hall.

Scolari, C. (2013). Narrativas Transmedia. Barcelona: Grupo Planeta.

Senge, P. (1999). La Quinta Disciplina: El arte y la práctica de la organización abierta al aprendizaje. Barcelona: Granica.

Shedroff, N. (1999). Information Interaction Design. A unified field theory of design en Jacobson, $R$ (Ed) Information Design. (1) pp. 267-292. Cambridge: MIT Press.

Simone, R. (2000). La Tercera Fase. Formas de saber que estamos perdiendo. Madrid: Taurus.

Strauss, A. y Corbin, J. (1990). Basicks of qualitative research techniques and procedures for developing grounded theory. Kluwer Academic Publishers: Human Sciences Pres.

Taylor, S y Bogan, R. (2000). Introducción a los métodos cualitativos en investigación. La búsqueda de los significados. España: Paidós

Tyack, D. y Cuban, L. (2001). En busca de la utopía. Un siglo de reformas de las escuelas públicas. México: Fondo de Cultura Económica.

Van Dijck, J. (2016). La Cultura de la Conectividad. Buenos Aires: Siglo XXI.

Vasilachis de Gialdino, I. (2006). Estrategias de Investigación Cualitativa. Barcelona: Gedisa.

Villa, A. (2000). Liderazgo y Organizaciones que aprenden. Actas del III Congreso Internacional sobre Dirección de Centros Educativos. Bilbao: ICE de la Universidad de Deusto: Mensajero.

Vincent, G. y Lahire, B. (2001). Sobre a historia e a teoría da forma escolar. Revista Educacao. Año XVI: Belo Horizonte: Facultade de Educacao. Universidad General de Minas Gerais (33). pp. 28 -44.

Vygotsky, L. (1987). Pensamiento y lenguaje: Teoría del desarrollo cultural de las funciones psíquicas. Buenos Aires: La Pléyade. 